\begin{tabular}{lcl}
\hline Bentham OPen & The Open Dentistry Journal & $\substack{\text { The } \\
\text { Open Dentisty } \\
\text { lournal }}$ \\
\hline CrossMark & Content list available at: www.benthamopen.com/TODENTJ/ \\
\hline
\end{tabular}

REVIEW ARTICLE

\title{
Ultralow Dose MSCT Imaging in Dental Implantology
}

\author{
Gerlig Widmann ${ }^{1, *}$ and Asma'a A. Al-Ekrish ${ }^{2}$ \\ ${ }^{I}$ Department of Radiology, Medical University of Innsbruck, Innsbruck, Austria \\ ${ }^{2}$ Department of Oral Medicine and Diagnostic Sciences, College of Dentistry, King Saud University, Riyadh, Saudi \\ Arabia
}

Received: November 17, 2017

Revised: December 20, 2017

Accepted: January 05, 2018

\section{Abstract:}

\section{Introduction:}

The Council Directive 2013/59 Euratom has a clear commitment for keeping medical radiation exposure as low as reasonably achievable and demands a regular review and use of diagnostic reference levels.

\section{Methods:}

In dental implantology, the range of effective doses for cone beam computed tomography (CBCT) shows a broad overlap with multislice computed tomography (MSCT). More recently, ultralow dose imaging with new generations of MSCT scanners may impart radiation doses equal to or lower than CBCT. Dose reductions in MSCT have been further facilitated by the introduction of iterative image reconstruction technology (IRT), which provides substantial noise reduction over the current standard of filtered backward projection (FBP).

\section{Aim:}

The aim of this article is to review the available literature on ultralow dose CT imaging and IRTs in dental implantology imaging and to summarize their influence on spatial and contrast resolution, image noise, tissue density measurements, and validity of linear measurements of the jaws.

\section{Conclusion:}

Application of ultralow dose MSCT with IRT technology in dental implantology offers the potential for very large dose reductions compared with standard dose imaging. Yet, evaluation of various diagnostic tasks related to dental implantology is still needed to confirm the results obtained with various IRTs and ultra-low doses so far.

Keywords: Radiation dose, Dental Implantology, Cone beam computed tomography, Multislice computed tomography, Ultralow dose, Filtered backward projection.

\section{INTRODUCTION}

Cone beam computed tomography (CBCT) and multi slice computed tomography (MSCT) are essential imaging modalities in dental implantology. The importance of cross-sectional imaging in visualizing the amount and shape of the edentulous ridge, and determining the position of neurovascular structures, nasal cavity, and maxillary sinuses relative to proposed implant sites is reflected by current guidelines for dental and maxillofacial radiology such as the position statement of the American Academy of Oral and Maxillofacial Radiology [1], European Association of Osseointegration (EAO) devised consensus guidelines [2] and the evidence-based guidelines from the European sedentex CT project [3]. Multiplanar cross-sectional and volume rendering 3D images may be obtained from MSCT

\footnotetext{
* Address correspondence to this author at the Department of Radiology, Medizinische Universitat Innsbruck, Innrain 52, Christoph-Probst-Platz, 6020 Innsbruck, Austria; Tel: +4351290030; E-mail: gerlig.widmann@i-med.ac.at
} 
datasets and used to assess implant sites and plan and guide treatment assisted by dedicated software and computerassisted fabrication of surgical guides.

However, the increasing use of cross-sectional imaging has become an increasing public and medical concern [4]. Radiologic societies have started awareness campaigns such as the Image Gently and Image Wisely Campaigns and the American College of Radiology dose index registry initiatives [5]. The European Union Council Directive 2013/59/Euratom of 5 December 2013 has set the basic safety standards for protection against the dangers arising from exposure to ionising radiation and requires a clear commitment to radiation protection. All doses due to medical exposures should be kept as low as reasonably achievable consistent with obtaining the required medical information; and the amount of patient exposure should form part of the report. This National Council on radiation protection has also attempted to emphasize the importance of optimization in medical imaging through modification of the ALARA As Low As Reasonably Achievable concept to ALADA, As Low As Diagnostically Achievable, which stresses that the aim of imaging should be to use the lowest possible dose which will produce diagnostic images [6]. The EU directive will have an impact on dental imaging and demands appropriate compliance by personnel involved in requesting, acquiring, and interpreting radiographic images. In addition, diagnostic reference levels for dental implant imaging should be developed and regularly reviewed.

The range of effective doses obtained from CBCT may show a broad overlap with MSCT. New generations of MDCT scanners have an enormous potential for dose reduction with ultralow dose imaging and may impart radiation doses equal to or lower than CBCT [7 - 9]. The purpose of this article is to review the current state of the art of ultralow dose MSCT imaging and Iterative Image Reconstruction Technology (IRT) in dental implantology imaging and to summarize their influence on spatial and contrast resolution, image noise, tissue density measurements, and validity of linear measurements of the jaws.

\section{ULTRALOW DOSE IMAGING}

There are many options for dose reduction including modification of basic imaging parameters, X-ray beam prefiltering, and application of IRT. Unfortunately, different MSCT vendors each have their own proprietary nomenclature for similar scanning factors, which may create confusion when comparing different scanners [5]. However, organizations must archive volume CT dose index $\left(\mathrm{CTDI}_{\mathrm{vol}}\right)$ and Dose Length Product (DLP) for every MSCT examination by series or anatomic area imaged. The CTDI $\mathrm{vol}_{\mathrm{v}}$ and DLP are estimates of average X-ray output from an acquired MSCT image series, which allows an easy comparison of the effect of different protocols, techniques and devices [10].

The most practical and most commonly used method to mitigate radiation exposure is to reduce tube voltage (kV) and effective tube current time product (mAs). High resolution reference protocols at $120 \mathrm{kV} / 100-200 \mathrm{mAs}$ with a $\mathrm{CTDI}_{\mathrm{vol}}$ of approximately $30 \mathrm{mGy}$ can be replaced by low dose protocols using $100-110 \mathrm{kV} / 50-80 \mathrm{mAs}^{\text {with a CTDI }} \mathrm{vol}_{\mathrm{v}}$ of approximately $10 \mathrm{mGy}[7,11]$. With reduced $\mathrm{mA}$, CT examination of the maxilla may be performed at an effective dose of $22 \mu \mathrm{Sv}$, which is comparable to that of a dental panoramic radiograph $(26 \mu \mathrm{Sv})$, while a CT scan of the mandible may be performed at an effective dose of $123 \mu \mathrm{Sv}$ which is comparable to a full-mouth survey with intra-oral films $(150 \mu \mathrm{Sv})$ [12]. Considering a scan length of $10 \mathrm{~cm}$ (i.e. a dentoalveolar scan of mandible \& maxilla), progressive reduction in the $\mathrm{kV}$ and $\mathrm{mAs}$ using ultralow dose protocols led to estimated effective doses of $87 \mu \mathrm{Sv}\left(\mathrm{CTDI}_{\mathrm{vol}} 4.14\right.$ mGy), $55 \mu \mathrm{Sv}\left(\mathrm{CTDI}_{\mathrm{vol}} 2.63 \mathrm{mGy}\right), 21 \mu \mathrm{Sv}\left(\mathrm{CTDI}_{\mathrm{vol}} 0.99 \mathrm{mGy}\right)$, and $11 \mu \mathrm{Sv}\left(\mathrm{CTDI}_{\mathrm{vol}} 0.53 \mathrm{mGy}\right)$, which are marked

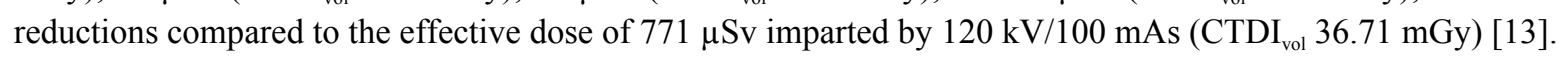

$\mathrm{X}$-ray tubes in MSCT usually allow voltages ranging from $140 \mathrm{kV}$ down to $80 \mathrm{kV}$ as the lowest possible tube potential. Recently, an X-ray tube providing $70 \mathrm{kV}$ has been tested by comparing $70 \mathrm{kV} / 75 \mathrm{mAs}\left(\mathrm{CTDI}_{\mathrm{vol}} 2.33 \mathrm{mGy}\right)$ with $\left.100 \mathrm{kV} / 40 \mathrm{mAs}_{(\mathrm{CTDI}} 3.95 \mathrm{mGy}\right)$ and $120 \mathrm{kV} / 40\left(\mathrm{CTDI}_{\mathrm{vol}} 6.31 \mathrm{mGy}\right)$ for imaging of paranasal sinus [14]. Image noise was significantly higher at lower doses; however, the signal noise ratio of soft tissues was increased at 70 $\mathrm{kV}$ and showed no significant difference to $100 \mathrm{kV}$ due to a higher organ attenuation of soft tissue structures with lower tube voltage settings [14]. Also, a 40\% decrease in CTDI $_{\text {vol }}$ and calculated effective dose was obtained with MSCT of the jaws when $\mathrm{kV}$ was reduced from 100 to 80, even though the $\mathrm{mA}$ was increased from 35 to 40 [9].

Another method for reducing MSCT dose is increasing the pitch. Pitch (beam pitch) is defined as the table movement per rotation/total thickness of all of the simultaneously acquired slices (total beam width $(n \times \mathrm{x})$, with $n$ slices and slice thickness T) [15]. The $\mathrm{CTDI}_{\mathrm{vol}}$ decreases linearly with increasing pitch, but the number of photons contributing to the images decreases also. A pitch of $\leq 1$ is typically used in MSCT of bone to decrease helical 
interpolation artifacts [15]. Using a second-generation dual source MSCT (combined use of a $90^{\circ}$ shifted X-ray tube) with large detector coverage for face and sinus imaging a pitch of 3 may provide sufficient diagnostic image quality compared with two single-source reference protocols with pitch 0.9 [16].

Prefiltration of the X-ray beam with a Tin filter has also been shown to reduce MSCT doses through constriction of

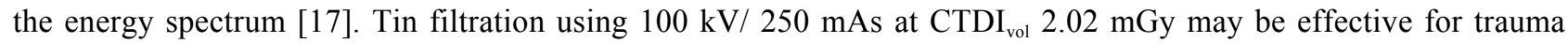
imaging and Tin filtration using $100 \mathrm{kV} / 150 \mathrm{mAs}$ at $\mathrm{CTDI}_{\mathrm{vol}} 1.22 \mathrm{mGy}$ may provide sufficient information at the lowest dose [17].

\section{ITERATIVE RECONSTRUCTION TECHNOLOGY (IRT)}

Recently, IRTs have been implemented to reduce image noise and improve quality of low dose images when compared with the traditionally used filtered back projection technique (FBP) [18]. Iterative reconstruction in image space (IRIS; Siemens Healthcare) includes an additional "correction loop" in which the obtained images are gradually approximated to their actual density distribution, thereby reducing image noise. The subjective image quality of a $60 \%$ dose reduced protocol at $120 \mathrm{kV} / 24 \mathrm{mAs}$ with IRIS may demonstrate no significant difference to a reference protocol using $120 \mathrm{kV} / 60 \mathrm{mAs}$ with FBP [19]. Sinogram-affirmed iterative reconstruction (SAFIRE; Siemens Healthcare) estimates the noise content in raw data by fluctuations in neighboring voxels and subtracts the noise stepwise in up to 5 repetitive correction loops. This technology may reduce image noise by $15 \%-85 \%$ [20].

Adaptive statistical iterative reconstruction (ASIR; GE Healthcare) used the images obtained from the FBP algorithm but integrates a comparison of the pixel values with an ideal value to selectively identify and then subtract noise from an image at adaptive blend levels, which can be selected typically from $10 \%$ to $100 \%$ [21]. Model Based Iterative Reconstruction (MBIR, VEO; GE Healthcare) uses a more complex system of prediction models. It does not rely on the FBP as a starting point but integrates the noise and the spatial and geometric features of the x-ray beam and detector technology [22]. Unfortunately, MBIR requires a considerably long computational time and, therefore, may not be practical for emergency imaging. Also, MBIR is not available with a bone kernel. In a human cadaver, ultralow dose images at $\mathrm{CTDI}_{\mathrm{vol}} 3.48 \mathrm{mGy}$, and $2.19 \mathrm{mGy}$ (ASIR-100), and $\mathrm{CTDI}_{\mathrm{vol}} 0.82 \mathrm{mGy}$ (MBIR) may provide similar subjective

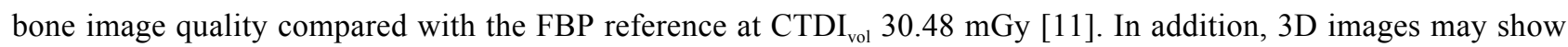
similar subjective image quality at $\mathrm{CTDI}_{\mathrm{vol}} 3.48 \mathrm{mGy}$ (all reconstructions), $2.19 \mathrm{mGy}$ (FBP and ASIR-100), 0.82 mGy (FBP, ASIR-100, and MBIR), 0.44 mGy (MBIR), and 0.22 mGy (MBIR) [23].

\section{SPATIAL RESOLUTION}

One study evaluated spatial and contrast resolution of ultralow dose images and ASIR and MBIR using the SedentexCT IQ Phantom (Leeds Test Objects Ltd., Boroughbridge, UK) [13]. As a general finding, reconstructions using the standard convolution kernel have a lower spatial resolution than those with bone kernel. Coronal sharpness is lower than axial sharpness, which reflects the non-isotropic image acquisition of MSCT. Also, with each reconstruction technique (FBP, ASIR, MBIR), lower doses produced images with lower spatial resolution. However, when a standard convolution kernel was used, ultralow dose protocols at $\mathrm{CTDI}_{\mathrm{vol}}$ of $4.14 \mathrm{mGy}$ and $2.36 \mathrm{mGy}$ combined with MBIR showed comparable spatial resolution to the standard clinical reference protocol at CTDI $_{\text {vol }}$ of 36.58 mGy using FBP; this may allow for a dose reductions of up to $93 \%$. However, ASIR was not found to significantly improve spatial resolution over FBP, regardless of the kernel used.

In a cadaver, dislocated craniofacial fractures were clearly detected with protocols using a CTDI ${ }_{\mathrm{vol}}$ as low as 1.0 mGy and non-dislocated fractures at a CTDI ${ }_{\mathrm{vol}}$ of $2.6 \mathrm{mGy}$, regardless of the reconstruction technique used. As such, due to their image smoothing effects, ASIR and MBIR did not improve fracture detection over FBP [24].

\section{CONTRAST RESOLUTION}

Reductions in dose increase noise and decrease contrast resolution. Approximately $80 \%$ reduction in dose with FBP demonstrated a markedly reduced CNR but no alteration of the modulation transfer function of two MSCT devices [25].

When evaluating contrast noise ratio (CNR) using the SedentexCT IQ Phantom imaged with ultralow dose MSCT and IRTs, MBIR may show the highest CNR and retain the highest relative CNR, followed by ASIR 100, ASIR 50 and

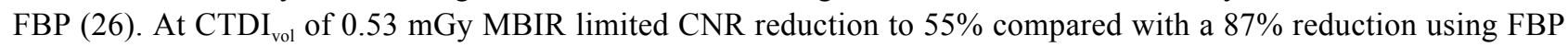
[26]. CNR using ASIR-100 may be better than ASIR 50 and FBP but the results seem to be not statistically significant [26]. 
In another study, when a bone kernel was used (except with MBIR) at a CTDIvol of $0.53 \mathrm{mGy}$ ( $98 \%$ of a standard dose), the use of ASIR and MBIR was found to reduce noise in images of the jaw bones compared to FBP; ASIR-50 reduced noise by $15 \%$, ASIR-100 by $24 \%$ and MBIR by $38 \%$. However, ASIR and MBIR did not appear to offer an advantage over FBP in this regards at higher doses [27]. Furthermore, the same study found that reconstruction technique had no significant effect on noise levels when a standard kernel was used with FBP and ASIR. On the other hand, studies of images of soft tissues and air obtained with standard kernels found that MBIR images demonstrated less noise than ASIR [11, 20, 28]. The contrasting results between images of bone and soft tissue may suggest that the effect of reconstruction technique and kernel on noise is not just affected by the dose level, but also by the type of tissue being imaged.

\section{BONE DENSITY MEASUREMENTS}

Bone density can be measured using the calibrated grey values as Hounsfield Units (HU), which are defined as linear transformations of measured X-ray attenuation coefficients of materials with reference to water [29]. The HU scale is based on two fixed values, which are $0 \mathrm{HU}$ for water and $-1000 \mathrm{HU}$ for air. Unlike CBCT, HU are relatively consistent across different MSCT scanners and HU calibration is an integrated part of quality control. Reduced MSCT doses are known to produce altered tissue densities within the images [30]. Evaluation of ultralow dose imaging using the SedentexCT IQ Phantom showed that HU values may vary significantly at very low doses [26].

Standard kernels may show less HU variability than bone kernels and ultralow dose protocols using FBP may show errors of up to $273 \mathrm{HU}$ [26]. Application of MBIR with standard kernel can reduce the error value to $138 \mathrm{HU}$ for the $\mathrm{CTDI}_{\mathrm{vol}}$ of $0.53 \mathrm{mGy}$ [26]. In human cadaver jaw bones, decreasing doses show an increase in mean HU of the jaw bones. Maximum mean differences in HU of 178.35 (bone kernel) and 273.74 (standard kernel) may be observed when comparing several ultralow-dose protocols with a reference protocol. Use of MBIR tends to show a lower discrepancy in HUs with ultra-low doses than the other reconstruction techniques. Using ultralow-dose protocols at a CTDI vol of 0.44 mGy and a standard kernel may demonstrate a difference of only 69.43 HU using MBIR compared with 162.48 HU using FBP. However, the same study did not detect a significant difference in density between the different reconstruction algorithms when a bone kernel was used [27].

\section{CLINICAL SIGNIFICANCE OF HU AND DENSITY WITH ULTRA-LOW DOSE MDCT}

The clinical impact of altered HU and noise profiles on implant imaging is still not completely clear. The image noise produced by a $90 \%$ reduction in dose combined with ASIR and MBIR did not significantly interfere with subjective image quality of 3D models [11]. But to fully understand how such ultra-low dose protocols may affect computer guided surgery, further studies on thresholding accuracy and CAD-CAM production of surgical guides need to be conducted.

The impact of the observed differences in noise and density between the various protocols on objective bone quality evaluation during implant site analysis is also still not clear. The HU obtained by standard dose MSCT has been correlated with primary implant stability, but there is still no reference standard to relate specific HU values to the determinants of implant site treatment planning and/or success [31 - 35]. Furthermore, since HU values vary with reductions in dose and reconstruction technique, any such standard, if developed, would only apply to images obtained with similar exposure protocols, especially the $\mathrm{kV}[30]$.

Regarding subjective classification of bone, the Lekholm and Zarb classification of bone assessed from standard dose MSCT images has been significantly correlated with subjective drilling resistance during implant osteotomies [36, 37]. Also, crestal cortical bone thickness assessed from standard dose MDCT has been strongly correlated with primary implant stability [32]. However, the changes in noise and HU seen with various combinations of low dose MSCT and reconstruction techniques, as well as the detrimental effect of MBIR on the visibility of thin bone may conceivably affect the subjective evaluation of bone [38]. Therefore, further studies are needed to relate the subjective appearance of bone from ultra-low dose images with the determinants of implant stability and success.

\section{VALIDITY OF LINEAR MEASUREMENTS OF THE JAWS}

Images obtained with MSCT at $\mathrm{CTDI}_{\mathrm{vol}} 2.5 \mathrm{mGy}$ may provide an acceptable accuracy of measurements for the purpose of maxillofacial surgery and image-based oral implant surgery [39]. With regards to linear measurements of dental implant sites recorded from sectional images, most combinations of ultra-low doses ranging from CTDI $_{\mathrm{vol}}$ 0.44-4.19 mGy combined with FBP, ASIR, and MBIR, demonstrated no systematic variation in linear measurements 
when compared with a standard dose FBP protocol $\left(\mathrm{CTDI}_{\mathrm{vol}}\right.$ 30.48-36.71 $\mathrm{mGy}$ ). The only combinations which demonstrated a statistically significant difference were within $\pm 0.1 \mathrm{~mm}(95 \% \mathrm{CI} \pm 1.15 \mathrm{~mm})$ of the reference measurements, which is not clinically significant [9].

Evaluating the influence of ultralow doses on Target Registration Error (TRE) of navigated surgery of facial and

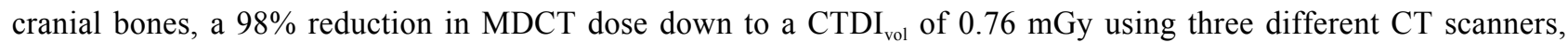
including one intraoperative scanner, showed no significant adverse effect on TRE [23].

\section{CONCLUSION}

Following public and medicolegal demands, efforts towards dose reduction have become an increasing responsibility in daily clinical routine. Implantologists and radiologists have to be aware that radiation dose of crosssectional imaging may vary substantially depending on scan parameters, including $\mathrm{kV}, \mathrm{mAs}$, pitch, collimation and others. Modern MSCT has an enormous potential for dose reductions and currently allows doses less than CBCT. Lowering MSCT doses increases noise and HU. But the noise can be markedly reduced with the application of IRTs. Ultralow dose images at $\mathrm{CTDI}_{\mathrm{vol}}$ markedly less than $1 \mathrm{mGy}$ did not significantly affect the height and width measurements of implant sites or TREs of navigated surgery. Further studies are still needed to investigate the effect of ultra-low doses and IRTs on other diagnostic tasks related to dental implantology such as visualization of the inferior alveolar nerve, accuracy of 3D segmentation and evaluation of influence on CAD-CAM produced surgical guides to confirm the results obtained so far. Last- generation CT scanners using ultralow dose technology and IRTs should be introduced in further clinical studies, in conformance with the goals of the Euratom directive. Implementation of ultralow doses in dental implantology may help in the formulation of DRLs to improve compliance with the ALADA concept.

\section{CONSENT FOR PUBLICATION}

Not applicable.

\section{CONFLICT OF INTEREST}

The authors declare no conflict of interest, financial or otherwise.

\section{ACKNOWLEDGEMENTS}

Declared none.

\section{REFERENCES}

[1] Tyndall DA, Price JB, Tetradis S, Ganz SD, Hildebolt C, Scarfe WC. Position statement of the American academy of oral and maxillofacial radiology on selection criteria for the use of radiology in dental implantology with emphasis on cone beam computed tomography. Oral Surg Oral Med Oral Pathol Oral Radiol 2012; 113(6): 817-26. [http://dx.doi.org/10.1016/j.oooo.2012.03.005] [PMID: 22668710]

[2] Harris D, Horner K, Gröndahl K, et al. E.A.O. guidelines for the use of diagnostic imaging in implant dentistry 2011. A consensus workshop organized by the European Association for Osseointegration at the Medical University of Warsaw. Clin Oral Implants Res 2012; 23(11): $1243-53$.

[http://dx.doi.org/10.1111/j.1600-0501.2012.02441.x] [PMID: 22432473]

[3] European Commission Radiation Protection No 172 Cone beam CT for dental and maxillofacial radiology (Evidence-based guidelines) Directorate General for Energy Directorate D-Nuclear Energy unit D4-Radiation Protection. 2012.

[4] United Nations Scientific Committee on the Effects of Atomic Radiation, Sources and effects of ionizing radiation Official Records of the General Assembly, Sixty-third Session, Supplement No 46. New York: United Nations 2008.

[5] Kalra MK, Sodickson AD, Mayo-Smith WW. CT radiation: Key concepts for gentle and wise use. Radiographics 2015; 35(6): 1706-21. [http://dx.doi.org/10.1148/rg.2015150118] [PMID: 26466180]

[6] National Council on Radiation Protection (NCRP): Achievements of the past 50 years and addressing the needs of the future Fiftieth annual meeting of the National Council on Radiation Protection and Measurements (NCRP) $2014 . \quad$ Available from: http://wwwncrponlineorg/Annual_Mtgs/2014_Ann_Mtg/PROGRAM_2-10pdf

[7] Jeong DK, Lee SC, Huh KH, et al. Comparison of effective dose for imaging of mandible between multi-detector CT and cone-beam CT. Imaging Sci Dent 2012; 42(2): 65-70. [http://dx.doi.org/10.5624/isd.2012.42.2.65] [PMID: 22783473]

[8] Ludlow JB, Timothy R, Walker C, et al. Effective dose of dental CBCT: A meta analysis of published data and additional data for nine CBCT units. Dentomaxillofac Radiol 2015; 44(1): 20140197. 
[http://dx.doi.org/10.1259/dmfr.20140197] [PMID: 25224586]

[9] Al-Ekrish AA, Al-Shawaf R, Schullian P, Al-Sadhan R, Hörmann R, Widmann G. Validity of linear measurements of the jaws using ultralowdose MDCT and the iterative techniques of ASIR and MBIR. Int J CARS 2016; 11(10): 1791-801. [http://dx.doi.org/10.1007/s11548-016-1419-y] [PMID: 27255542]

[10] Bauhs JA, Vrieze TJ, Primak AN, Bruesewitz MR, McCollough CH. CT dosimetry: Comparison of measurement techniques and devices. Radiographics 2008; 28(1): 245-53. [http://dx.doi.org/10.1148/rg.281075024] [PMID: 18203941]

[11] Widmann G, Schullian P, Gassner EM, Hoermann R, Bale R, Puelacher W. Ultralow-dose CT of the craniofacial bone for navigated surgery using adaptive statistical iterative reconstruction and model-based iterative reconstruction: 2D and 3D image quality. AJR Am J Roentgenol 2015; 204(3): 563-9.

[http://dx.doi.org/10.2214/AJR.14.12766] [PMID: 25714286]

[12] Homolka P, Gahleitner A, Kudler H, Nowotny R. A simple method for estimating effective dose in dental CT. Conversion factors and calculation examples for a clinical low dose protocol. RoFo Fortschr Geb Rontgenstr Nuklearmed 2001; 173(6): 558-62. [http://dx.doi.org/10.1055/s-2001-14991] [PMID: 11471298]

[13] Widmann G, Bischel A, Stratis A, et al. Spatial and contrast resolution of ultralow dose dentomaxillofacial CT imaging using iterative reconstruction technology. Dentomaxillofac Radiol 2017; 46(4): 20160452. [http://dx.doi.org/10.1259/dmfr.20160452] [PMID: 28059562]

[14] Bodelle B, Wichmann JL, Klotz N, et al. Seventy kilovolt ultra-low dose CT of the paranasal sinus: First clinical results. Clin Radiol 2015; 70(7): 711-5.

[http://dx.doi.org/10.1016/j.crad.2015.03.002] [PMID: 25912259]

[15] Goldman LW. Principles of CT: Multislice CT. J Nucl Med Technol 2008; 36(2): 57-68. [http://dx.doi.org/10.2967/jnmt.107.044826] [PMID: 18483143]

[16] Schell B, Bauer RW, Lehnert T, et al. Low-dose computed tomography of the paranasal sinus and facial skull using a high-pitch dual-source system--first clinical results. Eur Radiol 2011; 21(1): 107-12. [http://dx.doi.org/10.1007/s00330-010-1892-6] [PMID: 20644936]

[17] Lell MM, May MS, Brand M, et al. Imaging the parasinus region with a third-generation dual-source CT and the effect of tin filtration on image quality and radiation dose. AJNR Am J Neuroradiol 2015; 36(7): 1225-30. [http://dx.doi.org/10.3174/ajnr.A4270] [PMID: 25814658]

[18] Padole A, Ali Khawaja RD, Kalra MK, Singh S. CT radiation dose and iterative reconstruction techniques. AJR Am J Roentgenol 2015; 204(4): W384-92.

[http://dx.doi.org/10.2214/AJR.14.13241] [PMID: 25794087]

[19] Bulla S, Blanke P, Hassepass F, et al. Reducing the radiation dose for low-dose CT of the paranasal sinuses using iterative reconstruction: Feasibility and image quality. Eur J Radiol 2012; 81(9): 2246-50. [http://dx.doi.org/10.1016/j.ejrad.2011.05.002] [PMID: 21664084]

[20] Schulz B, Beeres M, Bodelle B, et al. Performance of iterative image reconstruction in CT of the paranasal sinuses: A phantom study. AJNR Am J Neuroradiol 2013; 34(5): 1072-6.

[http://dx.doi.org/10.3174/ajnr.A3339] [PMID: 23221946]

[21] Silva AC, Lawder HJ, Hara A, Kujak J, Pavlicek W. Innovations in CT dose reduction strategy: Application of the adaptive statistical iterative reconstruction algorithm. AJR Am J Roentgenol 2010; 194(1): 191-9. [http://dx.doi.org/10.2214/AJR.09.2953] [PMID: 20028923]

[22] Fleischmann D, Boas FE. Computed tomography-old ideas and new technology. Eur Radiol 2011; 21(3): 510-7. [http://dx.doi.org/10.1007/s00330-011-2056-z] [PMID: 21249371]

[23] Widmann G, Fasser M, Schullian P, et al. Substantial dose reduction in modern multi-slice spiral computed tomography (MSCT): Guided craniofacial and skull base surgery. RoFo Fortschr Geb Rontgenstr Nuklearmed 2012; 184(2): 136-42. [http://dx.doi.org/10.1055/s-0031-1281971] [PMID: 22274855]

[24] Widmann G, Dalla Torre D, Hoermann R, et al. Ultralow-dose computed tomography imaging for surgery of midfacial and orbital fractures using ASIR and MBIR. Int J Oral Maxillofac Surg 2015; 44(4): 441-6. [http://dx.doi.org/10.1016/j.ijom.2015.01.011] [PMID: 25680629]

[25] Suomalainen A, Kiljunen T, Käser Y, Peltola J, Kortesniemi M. Dosimetry and image quality of four dental cone beam computed tomography scanners compared with multislice computed tomography scanners. Dentomaxillofac Radiol 2009; 38(6): 367-78. [http://dx.doi.org/10.1259/dmfr/15779208] [PMID: 19700530]

[26] Widmann G, Bischel A, Stratis A, et al. Ultralow dose dentomaxillofacial CT imaging and iterative reconstruction techniques: Variability of hounsfield units and contrast-to-noise ratio. Br J Radiol 2016; 89(1060): 20151055. [http://dx.doi.org/10.1259/bjr.20151055] [PMID: 26859336]

[27] Widmann G, Al-Shawaf R, Schullian P, Al-Sadhan R, Hormann R, Al-Ekrish AA. Effect of ultra-low doses, ASIR and MBIR on density and noise levels of MDCT images of dental implant sites. Eur Radiol 2017; 27(5): 2225-34. [PMID: 27655306] 
[28] Botsikas D, Stefanelli S, Boudabbous S, Toso S, Becker CD, Montet X. Model-based iterative reconstruction versus adaptive statistical iterative reconstruction in low-dose abdominal CT for urolithiasis. AJR Am J Roentgenol 2014; 203(2): 336-40. [http://dx.doi.org/10.2214/AJR.13.11937] [PMID: 25055268]

[29] Pauwels R, Jacobs R, Singer SR, Mupparapu M. CBCT-based bone quality assessment: Are hounsfield units applicable? Dentomaxillofac Radiol 2015; 44(1): 20140238. [http://dx.doi.org/10.1259/dmfr.20140238] [PMID: 25315442]

[30] IST (National Institute of Science and Technology) Tables of x-ray mass attenuation coefficients and mass energy-absorption coefficients from $1 \mathrm{keV}$ to $20 \mathrm{MeV}$ for Elements $\mathrm{Z}=1$ to 92 and 48 additional substances of dosimetric interest. Last updated: May 19. 2015 Available from: http://wwwnistgov/pml/data/xraycoef/indexcfm

[31] Al-Ekrish AA. Bone Quality for Implants. In: Tamimi D, Ed, Specialty Imaging Dental Implants Altona: Elsevier. 2014.

[32] Merheb J, Van Assche N, Coucke W, Jacobs R, Naert I, Quirynen M. Relationship between cortical bone thickness or computerized tomography-derived bone density values and implant stability. Clin Oral Implants Res 2010; 21(6): 612-7. [http://dx.doi.org/10.1111/j.1600-0501.2009.01880.x] [PMID: 20666788]

[33] Turkyilmaz I, McGlumphy EA. Is there a lower threshold value of bone density for early loading protocols of dental implants? J Oral Rehabil 2008; 35(10): 775-81.

[http://dx.doi.org/10.1111/j.1365-2842.2008.01867.x] [PMID: 18482347]

[34] Turkyilmaz I, McGlumphy EA. Influence of bone density on implant stability parameters and implant success: A retrospective clinical study. BMC Oral Health 2008; 8: 32. [http://dx.doi.org/10.1186/1472-6831-8-32] [PMID: 19025637]

[35] Ikumi N, Tsutsumi S. Assessment of correlation between computerized tomography values of the bone and cutting torque values at implant placement: A clinical study. Int J Oral Maxillofac Implants 2005; 20(2): 253-60. [PMID: 15839119]

[36] Lee S, Gantes B, Riggs M, Crigger M. Bone density assessments of dental implant sites: 3 . Bone quality evaluation during osteotomy and implant placement. Int J Oral Maxillofac Implants 2007; 22(2): 208-12. [PMID: 17465345]

[37] Rokn A, Rasouli Ghahroudi AA, Daneshmonfared M, Menasheof R, Shamshiri AR. Tactile sense of the surgeon in determining bone density when placing dental implant. Implant Dent 2014; 23(6): 697-703. [PMID: 25347271]

[38] Hoxworth JM, Lal D, Fletcher GP, et al. Radiation dose reduction in paranasal sinus CT using model-based iterative reconstruction. AJNR Am J Neuroradiol 2014; 35(4): 644-9.

[http://dx.doi.org/10.3174/ajnr.A3749] [PMID: 24113467]

[39] Loubele M, Jacobs R, Maes F, et al. Radiation dose vs. image quality for low-dose CT protocols of the head for maxillofacial surgery and oral implant planning. Radiat Prot Dosimetry 2005; 117(1-3): 211-6. [http://dx.doi.org/10.1093/rpd/nci749] [PMID: 16461498]

(C) 2018 Widmann and Al-Ekrish.

This is an open access article distributed under the terms of the Creative Commons Attribution 4.0 International Public License (CC-BY 4.0), a copy of which is available at: (https://creativecommons.org/licenses/by/4.0/legalcode). This license permits unrestricted use, distribution, and reproduction in any medium, provided the original author and source are credited. 